Fifth International Conference on Sustainable Construction Materials and

Technologies. http://www.claisse.info/Proceedings.htm

\title{
CHARACTERIZATION OF FRESH CEMENTITIOUS MEDIA THROUGH WAVE DISPERSION
}

\author{
Sokratis Iliopoulos, Dimitrios G. Aggelis \\ Dept. of Mechanics of Materials and Constructions, Vrije Universiteit Brussel, Pleinlaan 2, \\ 1050 Brussels, Belgium
}

\begin{abstract}
Ultrasonics have been widely used for testing concrete in the hardened and fresh state. Serious advances have been made in the field throughout the last three decades in terms of wave analyses, hydration modeling while materials with various additives have been tested. However, from the ultrasonics point of view, most of the information is provided mainly by the traditional parameter of "pulse velocity" based on the initial waveform threshold crossing and secondary by the amplitude of the pulse. These features are very powerful, but their characterization capacity diminishes with the increasing heterogeneity of the material. Detailed measurements of phase velocity and attenuation as a function of frequency provide extended information since instead of a single pulse velocity value, they provide a whole curve of wave velocity or attenuation over the frequency range of interest. This allows to observe resonant peaks owing to the microstructure giving better possibilities for characterization and allowing the monitoring of the changes of the curve as the material evolves from viscous to solid. In this paper a summary of wave dispersion and attenuation measurements in cement paste and mortar is presented, supported by theoretical formulations considering the microstructure while challenges and potential improvements are discussed.
\end{abstract}

Keywords: Wave dispersion, concrete, phase velocity, attenuation.

\section{INTRODUCTION}

The quality control, material characterization, assessment and monitoring of the performance of fresh cement-based media [1-5] has been conducted for decades through elastic wave propagation. The reason is the direct connection of wave parameters to elastic properties, the numerous empirical correlations with strength and quality as well as the relatively easy application with commercial devices. The microstructure of heterogeneous media, however, can significantly distort the wave and diminish its amplitude. Despite of this disadvantageous effect, the frequency analysis of wave features (phase velocity and attenuation) can prove beneficial for monitoring purposes. Although the application and interpretation may be challenging in some cases, they reveal interesting information on the microstructure, they highlight the influence of each separate component on the overall performance [6] and lead to a more reliable characterization of cementitious materials [7,8]. Despite the huge number of studies concerning ultrasonic assessment of cementitious materials, only a small percentage of them treats the phenomenon of wave dispersion in the 
aforementioned material [indicatively 7-9]. As it has been shown, propagation through the material alters the shape of the signal in both the time and frequency domain. This distortion is different when concrete is fresh compared to hardened state. In the first case the trend of phase velocity-frequency curve is decreasing for frequencies beyond a low-frequency limit (around 100-150 kHz), while in the case of hardened, the opposite trend is observed with continuously increasing velocities before stabilizing at higher frequencies [7]. Therefore, taking also into account the delicate nature of the dispersion experiments in fresh cementitious materials, the latter should be complemented with theoretical studies and numerical simulations to shed light on the propagation conditions and confirm the experimental aspects.

Numerical simulations offer several advantages. They are fast and relatively easy to implement, they can be repeated numerous times without high additional cost and they can cover various complex geometrical cases. In addition, theoretical formulations that have proven their validity in treating wave propagation problems in heterogeneous or scattering media (for instance concrete) are also of great value. Due to the strong impedance mismatch between the stiff aggregates, the liquid fresh paste and air bubbles, scattering models have the potential to enlighten the reasons for the observed strong dispersion and attenuation trends. In the current study, all three tools are simultaneously used and efficiently combined to provide velocity vs frequency curves for fresh concrete.

\section{ULTRASONIC EXPERIMENTS}

The execution of the dedicated ultrasonic experiments is based on a waveform generator (Physical Acoustics Corporation WaveGen 1410), two broadband Panametrics V413 transducers of $500 \mathrm{kHz}$ center frequency arranged in a through transmission configuration. A PAC preamplifier 1220A is also used along with a special plexiglass-EPDM (ethylene propylene diene monomer) U-shaped mold (see Fig. 1). The design and execution are custom-based but geometric characteristics are based on Rilem recommendations [10]. The monitoring starts from the moment of casting of the cementitious material in the mold and is made possible due to the direct contact of the sensors with the investigated media through suitable machined holes. It is mentioned that the wave generator is of maximum amplitude of $10 \mathrm{~V}$, which is very low to the voltage of high energy commercial devices and therefore, it could not affect the microstructure and the hydration development of the material. Further details on the experimental setup can be found in recent literature [11]. 


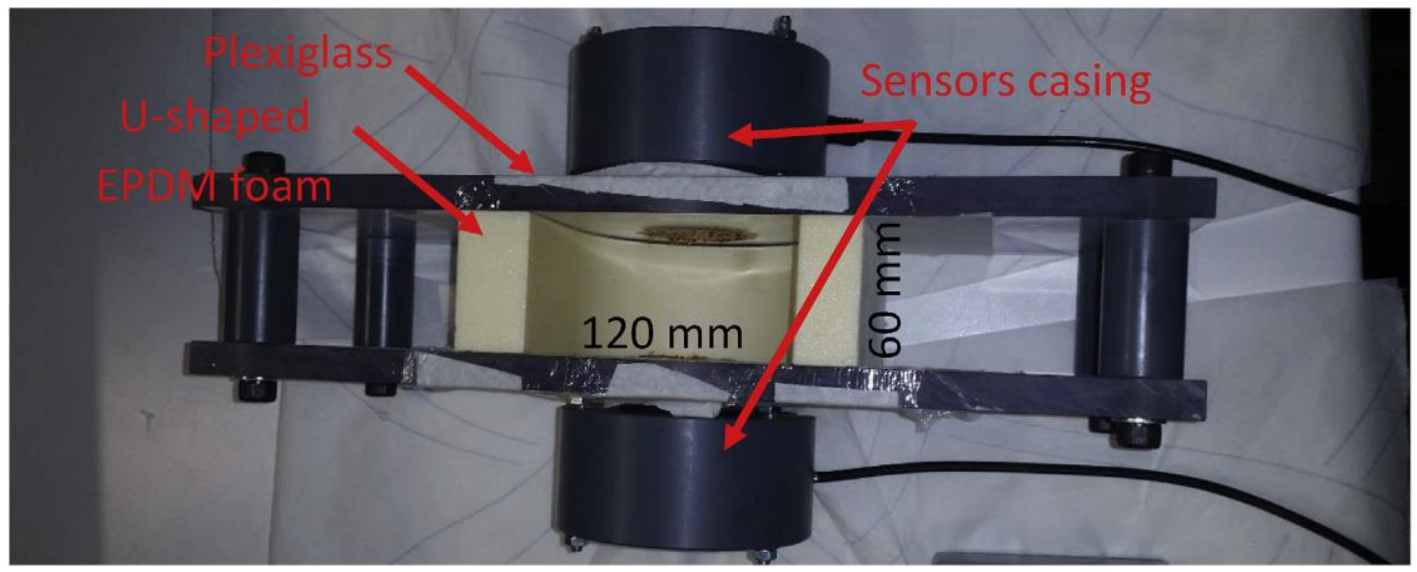

Fig. 1 Ultrasonic setup for fresh concrete testing.

\section{NUMERICAL ANALYSIS}

Parallel to the ultrasonic experiments a series of numerical simulations are performed using the Wave2000® software. The software operates by solving the two-dimensional (2D) acoustic (elastic and viscoelastic) wave equation based on the method of finite differences [12]. The geometry of the numerical model as well as the constituents of the fresh cementitious media and the boundary conditions are shown in Fig. 2. The density, Lame constants and the corresponding longitudinal and shear velocities of the cementitious matrix, the air and the inclusions are reported in Table 1. The volume content of air is indicatively selected equal to $1 \%$ based on laboratory measurements. The aggregate content varies between $30 \%$ and $40 \%$. The densities correspond to the real physical values of the material. The Lame constants of cement paste and air are calculated based on the corresponding wave velocities. The longitudinal and shear velocities of the aforementioned two mix constituents are reasonably selected based on literature values. More specifically, concerning the fresh cement paste the value of $1500 \mathrm{~m} / \mathrm{s}$ is considered for the longitudinal wave velocity, which is close to the one of water (in literature, velocity values for fresh cement paste vary from $300 \mathrm{~m} / \mathrm{s}$ to $2000 \mathrm{~m} / \mathrm{s}$ ). The shear velocity and the corresponding modulus in the same case is considered zero since the material is still in liquid form. Concerning the aggregates, the Lame constants are obtained from the manufacturer.

The two transducers are placed on the two sides of the medium in a through transmission arrangement similarly to the experiments. The transducer on the left serves as a pulser while the transducer at the right plays the role of the receiver (see Fig. 2). The excited signal has the shape of a sinus wave in sinusoidal envelope and it consists of five peaks. Different discrete frequencies are transmitted covering the range from $15 \mathrm{kHz}$ to $1 \mathrm{MHz}$. 


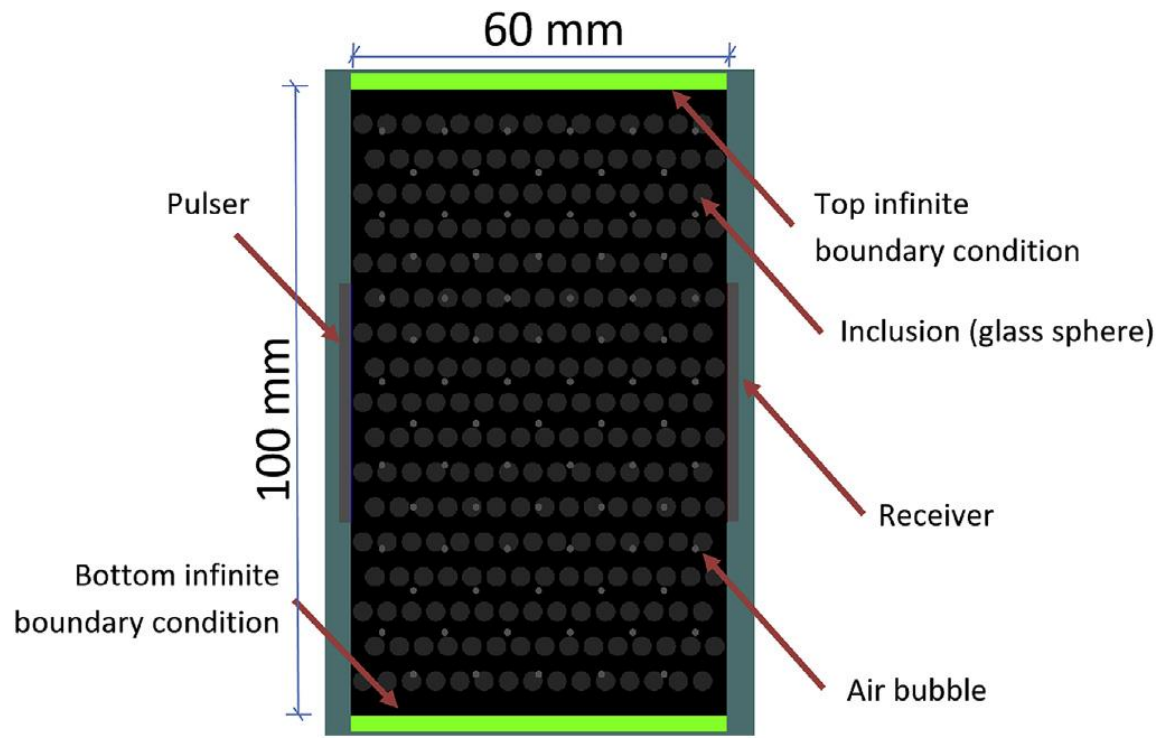

Fig. 2. Numerical model for wave propagation in fresh concrete

Table 1: Properties of fresh cementitious media constituents.

\begin{tabular}{|lccccc|}
\hline Mix constituents & $\rho\left[\mathrm{kg} / \mathrm{m}^{3}\right]$ & $\lambda[\mathrm{GPa}]$ & $\mu[\mathrm{GPa}]$ & $\mathrm{C}_{\mathrm{p}}(\mathrm{m} / \mathrm{s})$ & $\mathrm{C}_{\mathrm{s}}(\mathrm{m} / \mathrm{s})$ \\
\hline Cement paste & 1800 & 4.0 & 0 & 1500 & 0 \\
\hline Inclusions $(3 \mathrm{~mm})$ & 2460 & 26.0 & 33.2 & 6131 & 3674 \\
\hline Air $(1 \mathrm{~mm})$ & 1.2 & $142 * 10^{-6}$ & $1 * 10^{-11}$ & 344 & 0.091 \\
\hline
\end{tabular}

\section{SCATTERING MODEL}

Scattering models exhibit the benefit of simpler solution and less computational time than simulations. On the other hand, they have reduced flexibility on the parameters such as the shape and size distribution of scatterer. The scattering model employed in this study is the one of Waterman and Truell [13] which produces results very close to experimental in cases of heterogeneous media with strong impedance mismatch between the constituents. It is based on the solution of the wave scattering problem on a single scatterer embedded into a matrix, and application of the derived scattering amplitudes in the dispersion relation which takes into account the scatterer size and volume content and allows derivation of the complex wavenumber of the composite medium for any given frequency.

\section{RESULTS}

Indicative results of phase velocity vs. frequency will be shown in this section. Phase velocity is more sensitive to microstructural effects as shown previously in literature for cement paste and mortar [9]. Fig. 3a illustrates, among others, the experimental result of the phase velocity versus frequency curve for a fresh cement paste specimen with water-cement ratio of 0.55 . A sharp peak up to $9000 \mathrm{~m} / \mathrm{s}$ is noticed below $200 \mathrm{kHz}$, followed by a sudden drop to the stabilization phase velocity value of approximately $1700 \mathrm{~m} / \mathrm{s}$ for the rest of the frequencies. In the same figure, the result of the scattering model for two different volumetric 
contents of air bubbles is also depicted. The first case concerns $2.15 \mathrm{vol} \%$ cavities of mean size $1 \mathrm{~mm}$ in liquid matrix presenting also a peak below $200 \mathrm{kHz}$ and highlighting the nice qualitative and quantitative agreement with the experimental results. The horizontal line on Fig. $3 \mathrm{a}$ is the result of the scattering model for $0 \%$ air bubbles which naturally shows a constant velocity value. Fig. $3 \mathrm{~b}$ demonstrates the numerical simulation results for a similar problem (liquid cement paste matrix with properties reported in Table 1 and air bubbles of $1 \mathrm{~mm}$ ) with three different volumetric percentages of the air bubbles, namely $0 \%, 1 \%$ and $2.4 \%$. The results highlight the effect of air bubbles which imposes a local peak of phase velocity at relatively low frequencies. Noticing the qualitative and quantitative agreement between numerical simulations, the results of the scattering model and ultrasonic experiments it can be stated that the low frequency dispersion can safely be attributed to the air bubbles present in cement paste. In addition, given the strong sensitivity of the velocity peak to the air bubble content, this observation opens the way of more accurate characterization of the air content through the dispersion it causes for certain frequency bands. The above-mentioned combined results shed light into the mechanisms responsible for the wave phenomena exhibited in fresh cementitious media. The seemingly "irregular" phase velocity (which reaches values close to $9000 \mathrm{~m} / \mathrm{s}$ ) vs. frequency curves are attributed to the interaction of the waves with the cavities formed by the air bubbles. This is in accordance with studies in gels and suspensions where phase velocity exhibits a strong attenuation and phase velocity peaks at wavelengths much longer than the air bubble size [14].
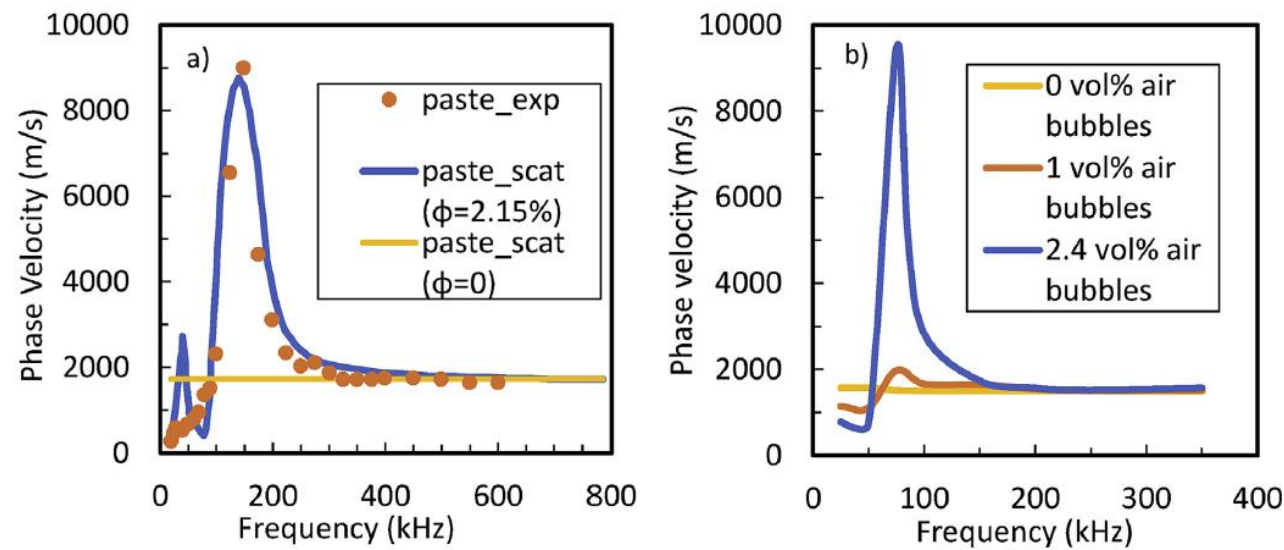

Fig. 3 Phase velocity vs. frequency curves from a) scattering theory and experiment, b) numerical simulation with different air bubble content.

\section{CONCLUSIONS}

The complicated nature of fresh cement-based materials calls for a more thorough investigation of the wave propagation in such heterogeneous media. In the current paper the synergy between the three different tools (ultrasonic experiments, numerical analyses and scattering theories) is presented. The results show a good match between the ultrasonic experiments, the numerical simulations and the analytical scattering model in terms of phase velocity vs. frequency curves. It is seen that air bubbles strongly influence the wave 
propagation due to strong impedance mismatch resulting in wave scattering. Attenuation features are also under investigation with the aim of detailed characterization of the microstructure of concrete and its development during setting.

\section{REFERENCES}

[1] Chung CW, Suraneni P, Popovics JS, Struble LJ. Using ultrasonic wave reflection to monitor false set of cement paste. Cement Concr Compos 2017 Nov 1;84:10-8.

[2] Trtnik G, Gams M. Recent advances of ultrasonic testing of cement based materials at early ages. Ultrasonics 2014 Jan 1;54(1):66-75.

[3] Kim G, Kim JY, Kurtis KE, Jacobs LJ. Drying shrinkage in concrete assessed by nonlinear ultrasound. Cement Concr Res 2017 Feb 1;92:16-20.

[4] Voigt T, Grosse CU, Sun Z, Shah SP, Reinhardt HW. Comparison of ultrasonic wave transmission and reflection measurements with $\mathrm{P}$-and $\mathrm{S}$-waves on early age mortar and concrete. Mater Struct 2005 Oct 1;38(8):729-38.

[5] Sayers CM, Dahlin A. Propagation of ultrasound through hydrating cement pastes at early times. Adv Cement Base Mater 1993 Oct 1;1(1):12-21.

[6] Nakahata K, Kawamura G, Yano T, Hirose S. Three-dimensional numerical modeling of ultrasonic wave propagation in concrete and its experimental validation. Construct Build Mater 2015 Mar 1;78:217-23.

[7] Aggelis DG, Polyzos D, Philippidis TP. Wave dispersion and attenuation in fresh mortar: theoretical predictions vs. experimental results. J Mech Phys Solid 2005 Apr 1;53(4):857-83.

[8] Chaix JF, Garnier V, Corneloup G. Ultrasonic wave propagation in heterogeneous solid media: theoretical analysis and experimental validation. Ultrasonics 2006 Feb 1;44(2):20010.

[9] Iliopoulos SN, Aggelis DG, Polyzos D. Wave dispersion in fresh and hardened concrete through the prism of gradient elasticity. Int J Solid Struct 2016 Jan 1;78:149-59.

[10] RILEM Technical Committee. Recommendation of RILEM TC 218-SFC: sonic

methods for quality control of fresh cementitious materials. Mater Struct $2011 \mathrm{Jul}$ 1;44(6):1047-62.

[11] S.N. Iliopoulos, L. De Smet, D.G. Aggelis, Investigating ultrasonic wave dispersion and attenuation in fresh cementitious materials: A combined numerical, analytical, and experimental approach, NDT \& E International, 100, 2018, 115-123

[12] Kaufman JJ, Luo G. A new dual-mode ultrasonic technique for assessing cortical bone. J Acoust Soc Am 2017 Oct;142(4):2565.

[13] Waterman PC, Truell R. Multiple scattering of waves. J Math Phys 1961 Jul;2(4):51237.

[14] Shukla SK, Elvira L. Comment on "Attenuation and dispersion of sound in dilute suspensions of spherical particles". J Acoust Soc Am 2000;108(1):126-46. The

Journal of the Acoustical Society of America. 2015 May;137(5):2962-5. 